\title{
P04-5-8 Poster session
}

\section{Microarray analysis in bleomycin-induced lung fibrosis}

\section{Yasuo Okamoto, Masahiro Komi, Yasuhiro Takenouchi, Kazuhito Tsuboi}

\section{Department of Pharmacology, Kawasaki Medical School, Japan}

Background: Lung fibrosis is a common consequence of many lung diseases and a leading cause of morbidity and mortality. The molecular mechanisms underlying the development of lung fibrosis remain poorly understood. We aimed to identify the genes associated with fibrogenesis using an Agilent microarray in a bleomycin-induced mouse model for lung fibrosis.

Methods: To induce lung fibrosis, mice were challenged with i.p. injections of bleomycin at $0.035 \mathrm{U} / \mathrm{g}$ twice weekly for 4 weeks. Microarray assays were performed to identify differentially expressed genes in lung tissues between bleomycinand saline-administered mice. To confirm differentially expressed genes, we determined their mRNA levels in the lung from bleomycin- or saline-administered mice using quantitative reverse transcription-PCR.

Results: In the lung from bleomycin-administered mice, 905 genes showed upregulation by more than 2.0-fold compared with the lung from saline-administered mice. In contrast, 732 genes showed downregulation by less than 0.5 -fold in the lung from saline-administered mice. Our results are consistent with previous results of well-documented gene expression. Interestingly, the expression of epiregulin (Ereg), growth differentiation factor 15 (Gdf15), and cyclin-dependent kinase inhibitors such as $C d k n 1 a$ and $C d k n 2 a$ was considerably induced in the lung after bleomycin treatment. Bleomycin also decreased the expression of Kruppel-like factor 15 (Klf15) and insulin-like growth factor binding proteins such as Igfbp3 and Igfbp6.

Conclusion: These data will aid in our understanding of fibrogenic mechanisms and contribute to the identification of candidate biomarkers of fibrotic disease development. 